\title{
El hombre relacional, comunicación y elementos estratégicos en el discurso de minas antipersonal, Colombiala una realidad
} Fecha de recepción: 16/08/2009 - Aprobación: 05/04/2010

\section{Mireya Barón Pulido}

\section{Resumen}

El presente artículo expone los resultados de la investigación que evidencian el comportamiento de elementos estratégicos en el discurso mediático sobre minas antipersonal en cuatro regiones de Colombia. Al hacer uso de la metodología cualitativa, se realizaron las siguientes fases: (1) análisis de contenido (AC) a través del software Qualrus/Atlas-ti en registros discursivos impresos y sonoros, (2) aplicación de instrumentos entrevistas intensivas a grupos focales con Dircom ONG en Bogotá y líderes regionales del país, y (3) triangulación de datos.

El proyecto contó con la colaboración del semillero de investigación constituido por cuatro estudiantes del Programa de Comunicación Social-Periodismo (Paloma Jiménez, Margarita Uribe, Pamela Zabala y Luis Palomino).

El respectivo cotejo de datos permite constatar el componente relacional del sujeto, toda vez que se interactúa en diversos escenarios según las necesidades a comunicar en una cultura de la prevención, del fortalecimiento y de la educación ante la situación vulnerable de las minas antipersonal en Antioquia, Cundinamarca, Nariño y Meta.

Los datos arrojados pretenden destacar que diseñar significados es, a la vez, crear realidades, propiciar acciones con propósitos específicos y lograr lecturas e interacciones simbólicas desde y para la educación, la prevención y el acompañamiento a la situación-conflicto.
Destacamos con esta investigación que la propuesta de una teoría de la estrategia de la comunicación, solo es viable en tanto se dinamice desde y para sujetos en escenarios específicos; pues es allí donde las necesidades comunicativas de los individuos perciben el discurso como acción, mas no como metalenguaje.

Para la actual investigación, el discurso-taller con los ciudadanos de la región, resulta ser la estrategia-acción adecuada y eficaz por excelencia, apoyada de registros mediáticos, como la producción sonora e impresa narrada para los usuarios actores de dicha realidad en diversas formas, según el nivel de conflicto.

\section{Abstract}

The present article exposes the results of the research that investigated the behavior of strategic elements in the media speech on anti-personnel landmines in four regions of Colombia. Using the qualitative methodology, the following phases were made: (1) Analysis of content (AC) through the Qualrus/Atlas-ti software in printed and sonorous discursive records; (2) Application of instruments, intensive interviews to focal groups with Dircom NGO in Bogota and regional leaders of the country; and (3) Data triangulation.

The project had the collaboration of the research seedbed made up by four students of the program of Social Communication Journalism (Paloma Jíménez, Margarita Uribe, Pamela Zabala, and Luis Palomino.) 
The respective check of information allows showing the relational component of the subject because everyone interacts in diverse stages according to the needs of reporting the prevention, the strengthening, and the education inside a culture before the vulnerable situation of the anti-personnel landmines in Antioquia, Cundinamarca, Nariño, and Meta.

The resulting data pretend to highlight that designing meanings is, at the same time, creating realities, causing actions with specific purposes, and achieving readings and symbolic interactions from and for education, prevention, and support to the conflict situation.

We emphasize with this research that the proposal of a communication strategy theory is only viable while it is stirred into action from and for subjects in specific stages since it is there where the communication needs of individuals perceive the speech as an action and not as metalanguage.

For the current research, the speech workshop with the citizens of the region turns out to be the suitable and effective strategic action supported by the media, such as the sonorous and printed production thought for the users who are actors of such reality in several ways, according to the conflict level.

\section{Palabras clave}

Estrategia, discurso acción, comunicación, significados.

\section{Key Words}

Strategy, action speech, communication, meanings.

\section{Introducción}

La sociedad del siglo XXI, la dinámica de múltiples y variadas interacciones entre los hombres y la tensión de los conflictos mundiales nos exigen repensar en toda su dimensión al hombre, desde su capacidad simbólica hasta su potencial del homo strategicus, tal y como lo caracteriza el español Rafael Pérez:

Un ser relacional que vive y se constituye como tal en sociedad y que se ve compe-

\footnotetext{
Reseña de autor

Mireya Barón Pulido

(Politécnico Grancolombiano)

mbaronpu@poligran.edu.co

Magister en Lingüística Española, Instituto Caro y Cuervo. Langue Française, ParisIV-Sorbonne. Docente Investigadora TC del Politécnico Grancolombiano, adscrita al Departamento de Comunicación. Líder del Gruplac Comunicación Estratégica y Creativa de la Facultad de Ciencias de Comunicación y Artes. Conferencista Internacional Conlacom-Bolivia 2007, Alaic-México 2008. Primer Congreso Internacional de Comunicaión Digital, Antioquia 2009. Publicaciones en Revistas Indexadas y PDF memorias en eventos nacionales e internacionales: "Semiosis en discurso minas antipersonal ", en Revista Electrónica Razón y Palabra № 69. Miembro de: Redicom (Red Colombiana de Investigación en Comunicación y Cultura), y de Cicre (Centro de Investigación Colombia Regional y Empresarial del Politécnico Grancolombiano).
}

lido a conjugar su presente y su futuro con otras personas, fuerzas y sistemas (Aguilar, 2008, párrafo 11).

La intención de un proceso comunicativo, la selección de canales mediáticos, los niveles de impacto que generan los mensajes y el cubrimiento de los mismos, forman parte de la percepción de una teoría desde la comunicación estratégica, redimensionada no desde el clásico management, sino desde la relación hombre-sociedad-comunicación.

El abordaje de realidades que afectan e impactan a la sociedad, con pluralidad de temas, y el carácter de interés humano, son especificidades de las ONG. (Pizzolante, 2007), campo de acción de la investigación en cuestión. En este sentido se entenderá una organización no gubernamental, a aquella cuya primordial intención es afectar a la comunidad civil ante la realidad de temas de orden social, que atentan con los derechos humanos del individuo tanto en su integridad personal como en su convivencia con los demás individuos de la sociedad.

En este orden de ideas, vale la pena reconocer la diversidad de tipos de interacción 
que fluyen en una organización, ya sea personal o institucional. El eje que dinamiza la gama de interacciones es el mismo acto comunicativo, visualizado en la figura 1.

\section{Figura 1}

\section{Niveles de comunicación en las organizaciones}

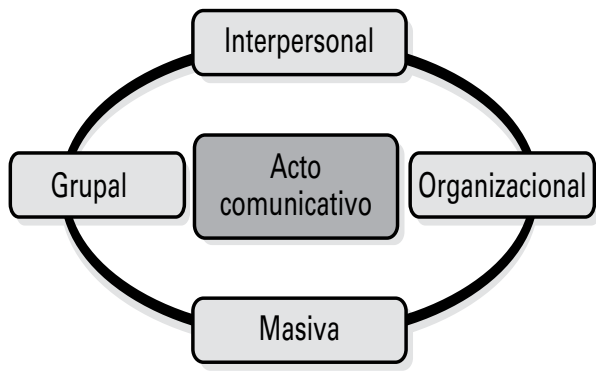

Fuente: elaboración propia
Analizar el comportamiento del sujeto (interpersonal), la interacción que se produce al interior de un grupo (grupal), la comunicación al interior de la misma organización (organizacional), y la relación entre información e impacto en sus audiencias (masiva), exigen delimitar un tipo de estudio desde la teoría estratégica de la comunicación.

Teniendo presente que uno de los aspectos posibles a estudiar en una organización son los niveles de comunicación, también resulta pertinente revisar el amplio recorrido que realiza y sigue el enfoque de dicha teoría. Desde Drucker, pasando por Porter hasta integrar la propuesta española de Pérez, se evidencia una preocupación por definir el alcance y papel de la comunicación en el ámbito, no solo de las organizaciones sino de la vida misma del hombre (tabla 1).

\section{Tabla 1}

Enfoques de la teoría estratégica*

\begin{tabular}{|l|l|l|}
\hline TEORÍA-ENFOQUE & REPRESENTANTE & \multicolumn{1}{c|}{ AXIOMA } \\
\hline \multirow{2}{*}{$\begin{array}{l}\text { Comunicación y } \\
\text { Hermenéutica }\end{array}$} & Sandra Masoni & $\begin{array}{l}\text { Diseñar y gestionar significados en acción } \\
\text { teniendo en cuenta decisiones, contexto y } \\
\text { proyectos. }\end{array}$ \\
\cline { 2 - 3 } & Rafónica yociocultural centrada en la situa- \\
\cline { 2 - 3 } & Competitividad & $\begin{array}{l}\text { Hombre relacional, dialogante, capacidad } \\
\text { humana en su discursiva simbólica. }\end{array}$ \\
\hline $\begin{array}{l}\text { Toería Neoclásica } \\
\text { Management }\end{array}$ & Peter Drucker & $\begin{array}{l}\text { La empresa competitiva desde competi- } \\
\text { dores, proveedores, compradores y pro- } \\
\text { ductos. }\end{array}$ \\
\hline
\end{tabular}

* Tabla compilada de diversas fuentes consultadas en portal de Foro Iberoamericano sobre Comunicación Estratégica (Fisec-Estrategias, Facultad de Ciencias Sociales de la Universidad Nacional de Lomas de Zamora, Argentina (2008) Año IV (9): 41-65 http://www.fisec-estrategias.com.ar/ (consulta: 6 de febrero de 2009).
La intención de un proceso comunicativo la selección de canales mediáticos, los niveles de impacto que generan los mensajes y el cubrimiento de los mismos, forman parte de la percepción de una teoría desde la comunicación estratégica, redimensionada no desde el clásico management, sino desde la relación hombresociedadcomunicación. 
La producción de mensajes se convierte en un proceso de semiosis, es decir en un proceso de construcción de significados y de sentidos. Es pertinente destacar que

la significación incorpora intrínsecamente la acción: aquellos efectos que producen y los que podría producir el objeto simbolizado, dentro de todas la circunstancias probables e improbables (Peirce, 1978: 48).
Por otro lado, dicho proceso no se realiza fuera de contexto. Sujetos que interactúan, objetos simbólicos que intervienen en la interacción, escenarios y tiempos en los que se desenvuelven afectan y dinamizan el proceso semiótico. Este tejido está a la vez inmerso en una cultura y, por ende, al interior de una organización. En la figura 2 se puede observar cómo cada nivel afecta al siguiente

Figura 2

Diseñar estrategias es diseñar significados, crear realidades

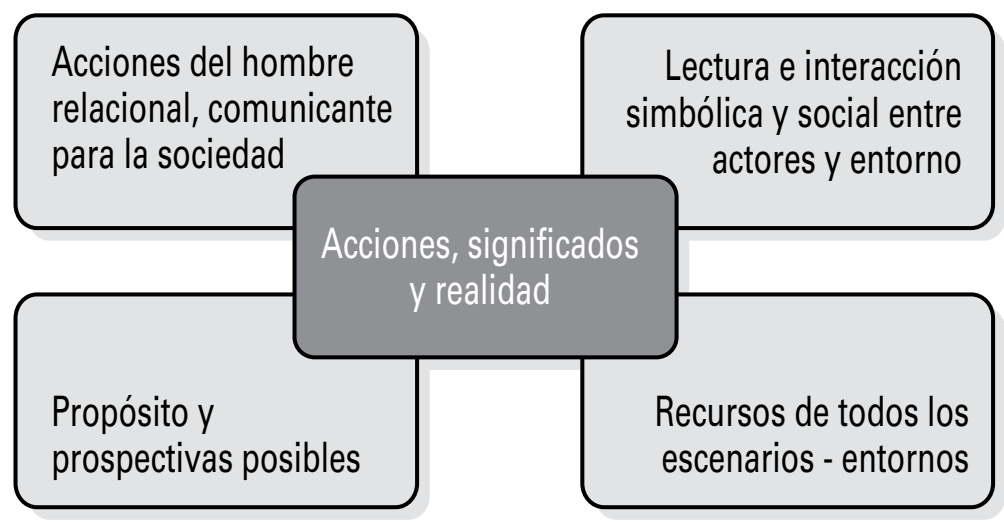

Fuente: elaboración propia

Como lo comenta Álvarez (2000: 4-5), La sociedad es generadora de mensajes, de propuestas... la comunicación permite que éstas lleguen a todos los sectores. Por tanto, la revolución del año 2000 es hacer de la comunicación una herramienta accesible para todos, en especial para aquellos sectores de la sociedad más vulnerables.

En estos términos, la comunicación, es percibida desde la interacción simbólica, tal y como lo advierte Pérez (2001: 427):

El signo está en lugar de la cosa representada, o en lugar de otro signo, lo que ya nos ha permitido de la mano de Baudrillard hablar del valor de uso de los signos y también del valor de cambio de los mismos como si de bienes o de mercancías se tratase.

Es precisamente desde el uso de signos, como la esfera de emisores, receptores y el mismo contenido del mensaje construyen realidades y acciones a través de mediaciones, tal y como lo comenta Pérez (2001: 444). No se trata de hacer el inventario de los medios como tal, sino de analizar el cómo se narran unos contenidos a través de los medios, llámense macromedios o micromedios; más aún, cómo se divulgan dichos discursos mediáticos y cómo estructuran niveles de cohesión al interior de una sociedad. 
En este orden de ideas, la apuesta de una comunicación estratégica que toma como punto de partida: (a) las miradas disciplinarias de la semiología, de la hermenéutica; y (b) los actores de la situación, la convierten en el enfoque pertinente para analizar el cubrimiento de realidades de riesgo en las sociedades actuales (tabla 2).

Tabla 2

Marco metodológico: análisis cualitativo

\begin{tabular}{|l|l|l|l|}
\hline \multicolumn{1}{|c|}{ TEMA } & \multicolumn{1}{|c|}{ UNIVERSO } & \multicolumn{1}{c|}{ MUESTRA } & \multicolumn{1}{c|}{$\begin{array}{c}\text { UNDAD DE } \\
\text { ANÁLISIS }\end{array}$} \\
\hline $\begin{array}{l}\text { Elementos estratégicos } \\
\text { en el discurso mediático } \\
\text { sobre el cubrimiento de } \\
\text { minas antipersonal en } \\
\text { Colombia. }\end{array}$ & $\begin{array}{l}\text { Discurso mediático } \\
\text { radial e impreso } \\
\text { en cuatro regiones } \\
\text { del país: Antioquia, } \\
\text { Cundinamarca, } \\
\text { Meta y Nariño. }\end{array}$ & $\begin{array}{l}\text { Cinco infomerciales, } \\
\text { boletines nacio- } \\
\text { nales-regionales, en } \\
\text { ediciones de 2005- } \\
2008 .\end{array}$ & $\begin{array}{l}\text { Referentes } \\
\text { y signos } \\
\text { (icónico, léxico, } \\
\text { cromático, } \\
\text { fonético), } \\
\text { sintaxis. }\end{array}$ \\
\hline
\end{tabular}

Fuente: Dominick - Wimmer, 2001.

Esta investigación cualitativa se desarrolló de manera escalonada por medio de las siguientes etapas: Momento A: diseño de instrumentos 1 y 2 a actores del proceso comunicativo, Momento B: AC análisis de contenido en el discurso impreso y sonoro con software cualitativo, y Momento 3: cotejo de datos entre fases 1 y 2 (tabla 3 ).

El diseño de instrumentos se realizó con base en la delimitación del marco conceptual que soportan las categorías a eviden- ciar en el discurso mediático. En el transcurrir de la pesquisa, se advirtió la necesidad de reagrupar las preguntas en tres dimensiones teniendo en cuenta la propuesta de una teoría de la comunicación estratégica humanística e integral: dimensión 1: organización; dimensión 2: comunicación; dimensión 3: discurso y contenido. En el siguiente acápite se destacan las percepciones de dichas entrevistas, soportadas en registro sonoro.
El diseño de instrumentos se realizó con base en la delimitación del marco conceptual que soportan las categorías a evidenciar en el discurso mediático.

Tabla 3

Resultados de la investigación

\begin{tabular}{|c|c|c|c|c|c|c|}
\hline \multicolumn{6}{|c|}{$\begin{array}{c}\text { MOMENTO A. INSTRUMENTO } 1 \text { PREGUNTA } \\
\text { DE LA ENTREVISTA INTENSIVA }\end{array}$} & $\begin{array}{l}\text { GRUPO FOCAL EMISORES } \\
\text { ONG BOGOTÁ }\end{array}$ \\
\hline \multicolumn{6}{|c|}{ 1. ¿Quiénes son los actores, partícipes del proceso comunicativo? } & \multirow{5}{*}{$\begin{array}{l}\text { Actores del proceso } \\
\text { comunicativo, involucra tanto } \\
\text { voces legítimas como voces } \\
\text { ciudadanas del común. Se } \\
\text { advierte la caracterización de un } \\
\text { tipo de ONG. }\end{array}$} \\
\hline & $\begin{array}{l}\text { Coordinadores } \\
\text { regionales }\end{array}$ & Victimas & ONG & Gobiemo & Comunidades & \\
\hline Manuel Gómez & $x$ & $x$ & $x$ & $x$ & $x$ & \\
\hline Camilo Serna & $X$ & $X$ & & & $X$ & \\
\hline Álvaro J. & $x$ & $x$ & & $x$ & & \\
\hline
\end{tabular}




\section{MOMENTO A. INSTRUMENTO 1 PREGUNTA DE LA ENTREVISTA INTENSIVA}

2. ¿Desde el contexto de la comunicación, cuáles cree usted que son las principales características de una $0 \mathrm{NG}$, y cuáles utilizan ustedes en su organización?

\begin{tabular}{|c|c|c|c|}
\hline & $\begin{array}{c}\text { Medios } \\
\text { alternativos }\end{array}$ & $\begin{array}{c}\text { Fuente imparcial de } \\
\text { información }\end{array}$ & $\begin{array}{c}\text { Organización que } \\
\text { acompaña a los } \\
\text { ciuddananos }\end{array}$ \\
\hline Manuel Gómez & $x$ & & \\
\hline Camilo Serna & & $x$ & \\
\hline Álvaro J. & & & $x$ \\
\hline
\end{tabular}

3. Los procesos comunicativos, tanto al interior como con el entorno de la organización, son de índole

\begin{tabular}{|c|c|c|c|c|}
\hline & Gerencial & Competitivo & Democerático & Otro \\
\hline Manuel Gómez & & $x$ & & \\
\hline Camilo Serna & & & & $x$ \\
\hline Álvaro J. & $x$ & $x$ & $x$ & \\
\hline
\end{tabular}

\section{GRUPO FOCAL EMISORES} ONG BOGOTÁ

Caracterización de ONG: es ante todo una entidad que vela por la ciudadanía, se visibiliza a través del uso de medios alternativos 0 locales.

Proceso comunicativo de $0 \mathrm{NG}$, tanto en su gestión interna como inter-regiones no se percibe de manera unificada. Inclusive no se le llega a identificar de manera precisa. Se percibe como un proceso que involucra tanto lo horizontal como lo vertical, en cuanto a interrelación Bogotá-regiones del país, Dircom-coordinadores regionales, facilitadores.
4. La labor comunicativa se caracteriza por.

\begin{tabular}{|c|c|c|c|c|c|}
\hline & $\begin{array}{c}\text { Diseñar } \\
\text { un plan de } \\
\text { medios }\end{array}$ & $\begin{array}{c}\text { Diseñar } \\
\text { mensajes para } \\
\text { la acción }\end{array}$ & $\begin{array}{c}\text { Administrar } \\
\text { recursos para la } \\
\text { comunicación }\end{array}$ & $\begin{array}{c}\text { Todas las } \\
\text { anteriores }\end{array}$ & Otras \\
\hline Manuel Gómez & & & & $X$ & \\
\hline Camilo Serna & & $X$ & $X$ & $X$ & $X$ \\
\hline Álvaro J. & $X$ & $X$ & & $X$ & \\
\hline
\end{tabular}

Actividad como gestión de comunicación, se percibe como un todo integral y dinámico, que involucra desde diseño de plan de medios hasta la administración de recursos. Inclusive se destaca, promover la praxis de la reportería en la ciudadanía del común.

Fuente: elaboración propia 


\section{MOMENTO A. INSTRUMENTO 1 PREGUNTA} DE LA ENTREVISTA INTENSIVA

5. ¿Quiénes son los usuarios de los mensajes o tienen diversos perfiles de usuarios según el tipo de medio-canal?

\begin{tabular}{|c|c|c|c|c|c|c|c|}
\hline & $\begin{array}{c}\text { Todos } \\
\text { los } \\
\text { actores }\end{array}$ & $\begin{array}{c}\text { Medios } \\
\text { nacionales } \\
\text { eimpresos }\end{array}$ & $\begin{array}{c}\text { Agencias } \\
\text { guthennamen- } \\
\text { tales }\end{array}$ & $\begin{array}{c}\text { Nlaciones } \\
\text { unidias }\end{array}$ & ONIG & $\begin{array}{c}\text { Varios púthicos } \\
\text { (Impresos, } \\
\text { cartillas, TV, } \\
\text { Intennet) }\end{array}$ \\
\hline Manuel Gómez & & & & & & & $X$ \\
\hline Camilo Serna & & $X$ & $X$ & $X$ & $X$ & $X$ & \\
\hline Álvaro J. & $X$ & & & & & & \\
\hline
\end{tabular}

6. ¿Cómo es el proceso de divulgación de los mensajes en las regiones-ciudades?

\begin{tabular}{|c|c|c|c|c|c|c|}
\hline & Web & Entrevistas & $\begin{array}{c}\text { Difiusión en } \\
\text { comunidades }\end{array}$ & $\begin{array}{c}\text { Elementos } \\
\text { comunicativos }\end{array}$ & Coordinadores & $\begin{array}{c}\text { Monitor de } \\
\text { minas }\end{array}$ \\
\hline Manuel Gómez & $X$ & & & & $X$ & $X$ \\
\hline Camilo Serna & & & & $X$ & $X$ & \\
\hline Álvaro J. & $X$ & $X$ & $X$ & & & \\
\hline
\end{tabular}

7. En las acciones de la organización han realizado un monitoreo del IMPACTO causado por las campañas en las regiones del país (existe algún registro)?

\begin{tabular}{|c|c|}
\hline & Nlo existe \\
\hline Manuel Gómez & $x$ \\
\hline Camilo Serna & $x$ \\
\hline Álvaro J. & $x$ \\
\hline
\end{tabular}

8. Las expresiones-enunciados utilizados en las campañas tienen el carácter de:

\begin{tabular}{|c|c|c|c|c|c|}
\hline & Informar & Prevenir & $\begin{array}{c}\text { Educar- } \\
\text { Alfabetizar }\end{array}$ & $\begin{array}{c}\text { Reforzal } \\
\text { actitudes }\end{array}$ & $\begin{array}{c}\text { Plan padino a } \\
\text { víctimas }\end{array}$ \\
\hline Manuel Gómez & $X$ & $X$ & & $X$ & \\
\hline Camilo Serna & $X$ & $X$ & & $X$ & \\
\hline Álvaro J. & $X$ & $X$ & $X$ & $X$ & $X$ \\
\hline
\end{tabular}

GRUPO FOCAL EMISORES ONG BOGOTÁ

Se percibe el interés por cubrir e interactuar con todos los actores de la situación conflicto; a tal punto que exige adecuar el medio a través del cual es más efectivo el mensaje según región.

Se debe aclarar que la web está en construcción. Sin embargo es vital el rol de divulgación del mensaje por medio de los coordinadores, en cada región, mediante actividades tipo charlas y talleres.
Acción vital se implementará en el plan de acción y de medios: monitoreo en regiones. Ello permitirá constatar el nivel de comunicación y efectos en la población receptora. La situación de conflicto armado dificulta esta faceta.

Es vital la intención y acción de prevención, reforzar actitudes y Plan Padrino.

En este caso, no se trata de informar por informar. Es generar interacción simbólica desde estos tres niveles específicos. 


\section{MOMENTO A. INSTRUMENTO 1 PREGUNTA} DE LA ENTREVISTA INTENSIVA

9. Los mensajes emitidos en las campañas cumplen con los siguientes aspectos:

\begin{tabular}{||c|c|c|c|c|c|}
\hline & Informar & Prevenir & $\begin{array}{c}\text { Elucar- } \\
\text { Alfabetizar }\end{array}$ & $\begin{array}{c}\text { Reforzar } \\
\text { actitudes }\end{array}$ & $\begin{array}{c}\text { Plan padrino a } \\
\text { víctimas }\end{array}$ \\
\hline Manuel Gómez & $X$ & $x$ & & $x$ & \\
\hline Camilo Serna & $X$ & $x$ & & $x$ & \\
\hline Álvaro J. & $x$ & $x$ & $x$ & $x$ & $x$ \\
\hline
\end{tabular}

GRUPO FOCAL EMISORES ONG BOGOTÁ

El diseño de campaña (radial-impresa) constata el ítem anterior. La estrategia es prevención, reforzar actitudes y Plan Padrino.
10. La comunicación visual de la campaña (fotografía), narra: (a). la situación conflicto, (b). la situación prevención:

\begin{tabular}{|c|c|c|}
\hline & La situación conflicto & La situación prevención \\
\hline Manuel Gómez & $X$ & $X$ \\
\hline Camilo Serna & $X$ & $X$ \\
\hline Álvaro J. & $X$ & $X$ \\
\hline
\end{tabular}

Colombia en 2009, exige que se le acompañe desde una comunicación mediática cuya acción desde la estrategia, no olvide la existencia de la minas antipersonal sentida como conflicto, a la vez que vivida como prevención.

Fuente: elaboración propia

A continuación, se compilan los gráficos que registran los resultados del instrumento 2, aplicado a líderes regionales. Los recuadros en gris corresponden a la pre-

gunta realizada (figura 3).

\section{Figura 3}

Respuestas líderes regionales

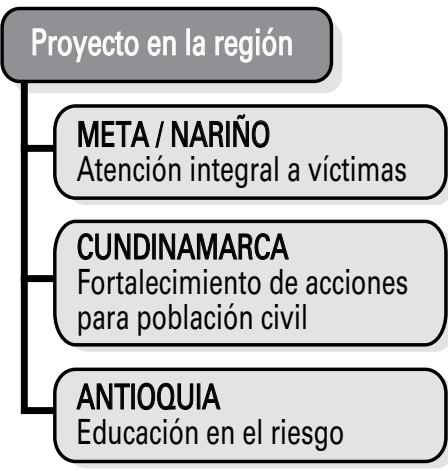

Misión del proyecto

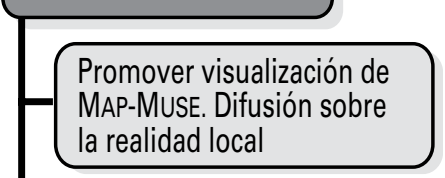

Promover visualización de problema y abogacía de acciones locales

Fuente: elaboración propia 

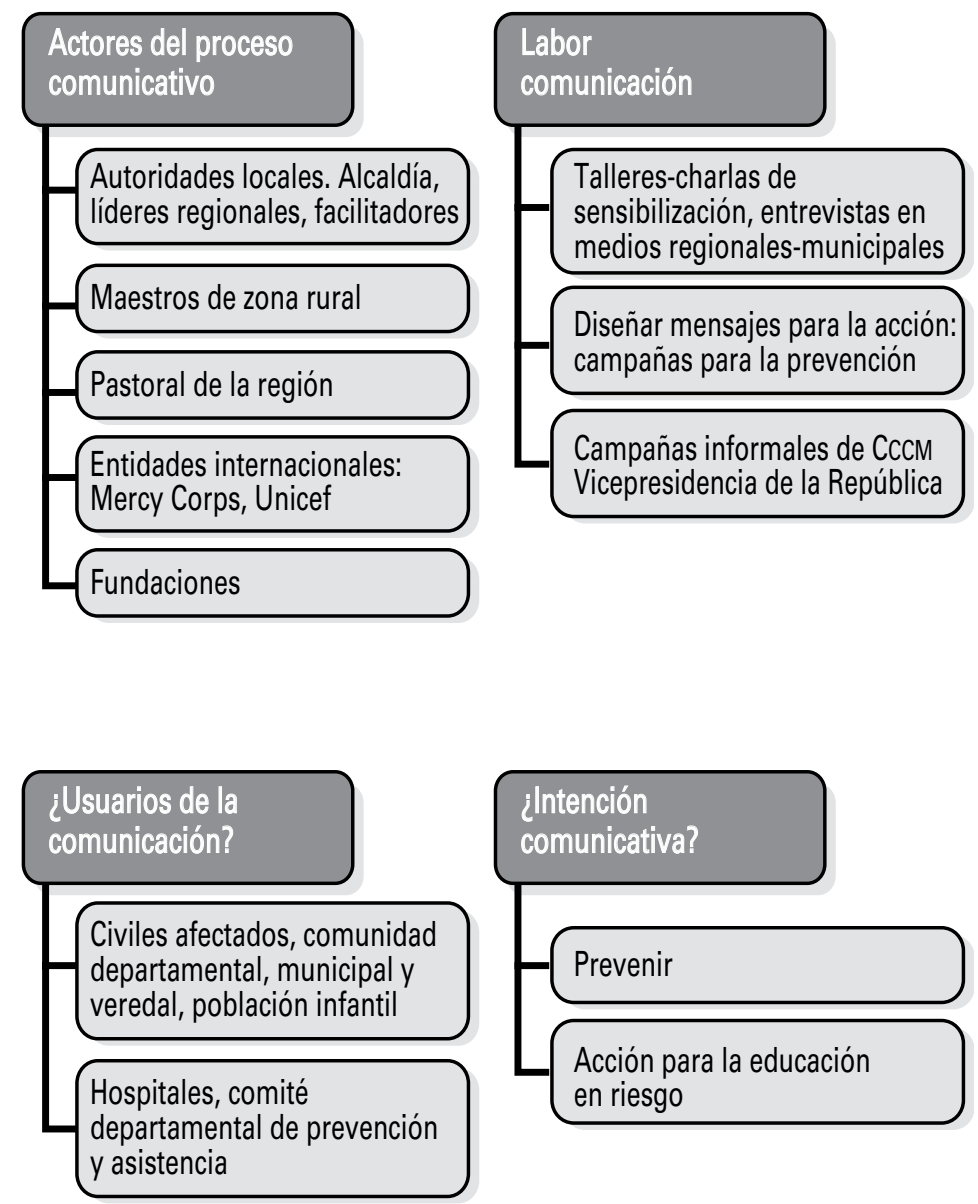

Fuente: elaboración propia

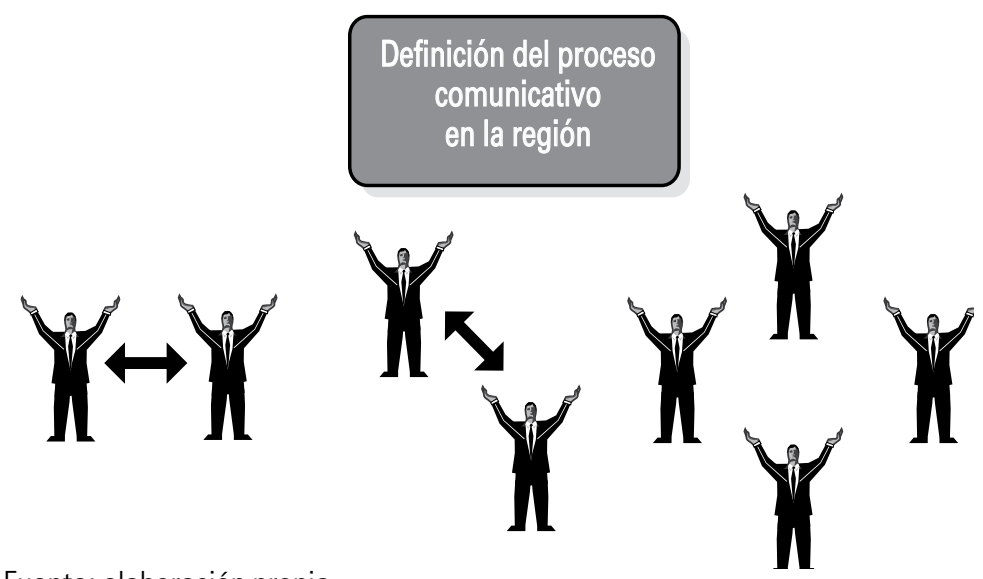

Fuente: elaboración propia 
Junto a estas respuestas, a continuación se visualizan las redes semánticas de la tendencia de teoría de comunicación estratégica que sugieren la dinámica comunicativa de la praxis discursiva sobre minas antipersonal, teniendo en cuenta: los supuestos

\section{Figura 4}

Caracterización teoría comunicación estratégica en discurso

\section{minas antipersonal}

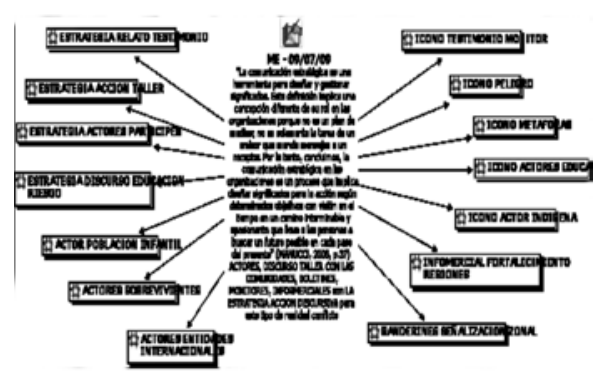

Fuente: elaboración propia

\section{Figura 6}

Estrategia comunicación visual en impresos

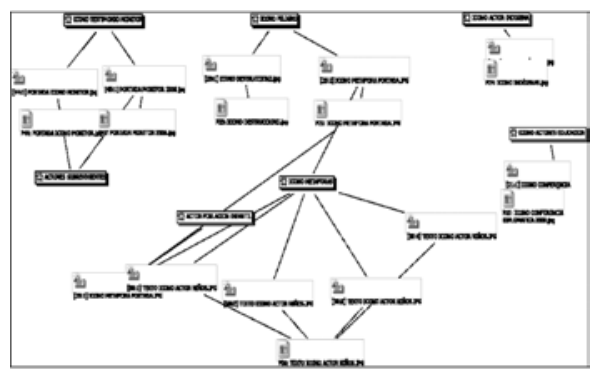

Fuente: elaboración propia desglosados a lo largo del texto, el análisis de contenido en impresos e infomerciales elaborados por la ONG y los datos arrojados por los dos instrumentos aplicados (figuras 4 al 7).

\section{Figura 5}

Estrategia acción taller

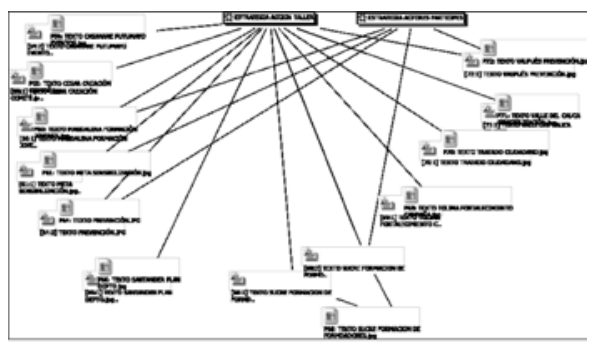

Fuente: elaboración propia

\section{Figura 7}

Estrategia comunicación sonora y actores

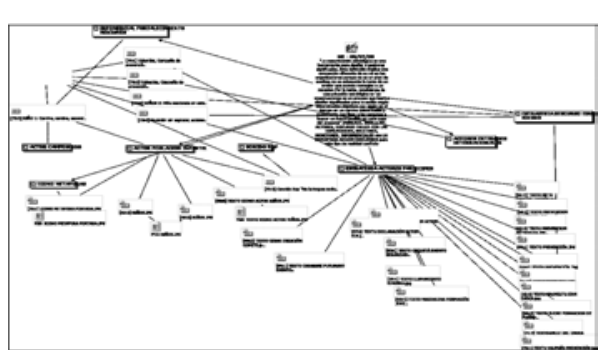

Fuente: elaboración propia

Nota editorial: Para ver las imágenes en el tamaño adecuado diríjase a las siguiente dirección: http://www.poligran.edu.co/cicre/elhombrerelacional.pdf. 


\section{Conclusiones}

La elaboración de significados le exigen al comunicador del siglo XXI, redimensionar el quehacer en todos los componentes del individuo, es decir a través de la esfera axiológica y hermenéutica de los procesos comunicativos.

En la investigación compilamos la tendencia de las percepciones que tienen los diversos actores que trabajan en pro de una comunicación preventiva o de fortalecimiento en las regiones objeto de estudio.

Se supera el plan de medios. Una comunicación estratégica exige la capacidad para interactuar acorde con las expectativas y necesidades del entorno sociocultural: el uso de acciones discursivas como el relato, el testimonio, el taller, el infomercial solo son viables e interpretables cuando se conoce de manera cercana el usuario real de dicha realidad: poblaciones campesina, infantil, indígena. El conjunto de actores partícipes de este proceso y su labor comunicativa ante contextos de alta vulnerabilidad, nos permiten sustentar que es en la acción-interacción como se elaboran y se interlocutan discursos que superan el metadiscurso, y que se convierten en el actor relacional del proceso comunicación-educación.

En las sociedades de la región de América Latina e Iberoamérica, situaciones de alta vulnerabilidad le demandan al comunicador actuar, estrategar -en términos del Maestro Español Rafael Pérez-, siempre teniendo presente: actores con sus índices de alfabetización, lugares, tiempos y contextos específicos comunicativos, por tanto, la selección de relatos, de actores partícipes, de iconicidad y de acciones discursivas son todo un reto en este tipo de procesos

\section{Bibliografía}

1. Aguilar, R., de Lourdes, M. (2008). Estrategia, ¿la misma para todos? FisecEstrategias - Facultad de Ciencias Sociales de la Universidad Nacional de Lomas de Zamora, VI (9), 41-65, 20 de mayo. [Disponible en:] http://www.cienciared.com. ar/ra/doc.php? $\mathrm{n}=891$. (consulta: el 18 de febrero de 2009).

2. Alvárez, E., Icaza Longoria. (2000). Medios y sociedad civil: una alianza para el control político (mayo). [Disponible en:] http://www.razonypalabra.org.mx/ anteriores/n18/18ealvarez.html. (consulta: el 2 de marzo de 2009).

3. Dominick - Wimmer. (2001). Introducción a la investigación de medios masivos de comunicación. México: Thompson.

4. Idea Works. (2002). Qualrus Sofyware Manual The Intelligent Qualitative Analysis Program. Columbia.

5. López, Daniel Fernando. (2006). Humanizar la comunicación, la mejor apuesta de la organización. En Varios autores. Comunicación empresarial, plan estratégico como herramienta gerencial. Bogotá: Centro de Investigaciones de la Comunicación Corporativa Organizacional (Cicco), ECOE, Universidad de la Sabana, 17-24.

6. Manucci, M. (2008). Complejidad, incertidumbre y estrategia. Hipótesis y desafíos para transitar la inestabilidad del contexto actual. Presentado en Fisec-Estrategias, Facultad de Ciencias Sociales de la Universidad Nacional de Lomas de Zamora, 1 (10), 
3-43. [Disponible en:] http://www.cienciared.com.ar/ra/usr/9/656/fisec_estrategias_ n10_pp3_43.pdf. (consulta: 17 de febrero de 2009).

7. Moya, Isabel. (2008). Otredad y cohesión social: una reflexión desde los medios, las imágenes y el imaginario. Ponencia presentada en VIII Congreso Iberoamericano de Género y Comunicación en La Habana (Cuba) (mayo). [Disponible en:] http:// www.webislam.com/pdf/pdf.asp?idt=10807. (consulta: 3 de marzo de 2009).

8. Peirce, Charles S. (1978). Lecciones sobre el pragmatismo. Aguilar

9. Pereira, J. M., Burbano, L. (2002). Educación superior e investigación en comunicación en Colombia. Revista Escribanía, 8. Manizales: Universidad de Manizales

10.Pérez, Alberto. (2004). Podemos hacer mejor las cosas: Hacia una nueva teoría estratégica refundada desde la comunicación. En Revista Interacción, 36-40 (Colombia) (mayo). [Disponible en:] www.communicationforsocialchange.org. (consulta: 25 de agosto de 2008).

11.Pizzolante N., Italo. (2007). Adecuarse al nuevo entorno empresarial o asumir el desafío de modelarlo. En Revista Signo y Pensamiento, XXVI (51), 169. Bogotá: Pontifica Universidad Javeriana.

12.Ulloa T., Cesar. (2008). Comunicación para el desarrollo: encrucijadas y perspectivas, América Latina en movimiento. [Disponible en:] http://alainet.org/ active/22047\&lang=es. (consulta: 01de febrero de 2009).

\section{Muestras discursivas}

1. ONG Campaña Colombiana Contra Minas, (CCCM) (2007), Infomerciales,

2. Unesco, ONG, CCCM (2007), Cartilla Memorias.

3. ONG, CCCM (2008), Boletín Informativo Regional.

4. ONG CNRR (2008), Boletín Abril.

5. ONG CCCM $(2005,2006,2007)$, Monitor.

\section{Fuentes primarias}

1. Navas Luz Estella - Líder Regional Cundinamarca

2. Rojas Lina Paola - Líder Regional Meta

3. Jiménez Olga Lucia - Líder Regional Antioquia

4. Cabrera T. Álvaro Fernando - Líder Regional Nariño

5. Manuel A. Gómez - Ángela Sanabria - Dircom CCCM Bogotá

6. Camilo Serna - Director Encargado CCCM

7. Álvaro Millán - Coordinación Nacional CCCM 